\title{
Mean Field Annealing Deformable Contour Method: A Constrained Global Optimization Approach
}

\begin{abstract}
This paper presents an efficient constrained global optimization approach based on mean field annealing (MFA) theory to the problem of contour energy minimization with a contour interior constraintfor object boundary extractions. In the method, with a given contour energy function, different target boundaries can be modeled as constrained global optimal solutions under different constraints expressed as a set of parameters characterizing the target contour interior structures. To search for the constrained global optimal solutions, a fast and efficient global approach based on MFA is employed to avoid local minima, which has been very difficult to achieve in most deformable contour methods. As an illustrative example, three target boundaries in a synthetic image are modeled as constrained global energy minimum contours with different constraint parameters and are successfully located using the derived algorithm. A conventional variational based deformable contour method [1] with the same energy function and constraint fails to achieve the same task. Experimental evaluations and comparisons with other methods on ultrasound pig heart, MRI knee, and CT kidney images where gaps, blur contour segments having complex shape and inhomogeneous interiors have been conducted with most favorable results.
\end{abstract}

Keywords: Snakes, curve evolution, variational based approach, level set, and mean field annealing.

\section{Introductions}


Image segmentation is a fundamental issue in computer vision. Energy based approach [22] [23] [2] may be one of the most influential image segmentation approaches. The basic idea of energy based methods is to model some global image properties [22] that capture the characteristics of the target regions/boundaries into energy functions; and then retrieve these target regions/boundaries via an energy minimization process. There are two important factors in most energy based methods: energy function formulations and energy minimization approaches. Energy functions may be classified as image based energy functions [22], for segmenting an entire image, and contour/region based energy, for object segmentations [2] [4]. Given an energy formulation, energy minimization approaches are then to minimize energy for retrieving the desired energy minimum. Generally, most energy minimization approaches are local methods [2] [4]. Since local energy minima can be arbitrarily far from the global optimum, these approaches may produce results that may not convey any of the global image properties encoded in energy functions. Some global approaches including simulated annealing 23] [14] [15], graph theory [22], and deterministic annealing 21] have also been proposed with rather successful results. Simulated annealing approaches often demand a high computational complexity [23] [14]. To reduce the computational complexity, faster temperature parameter decreasing scheme are used but may produce local energy minimum solutions that may be far from the global optimal solutions [24]. Graph theory based image segmentation methods provide a more efficient global approach for some energy functions [22]. Most of these methods [22] are used for image classification and are difficult in incorporating object shape information [25]. Deterministic annealing [26] has been successfully applied for data clustering problems and is further extended for texture image classification applications [21]. In this paper, we apply mean field annealing approach (MFA), which is a deterministic annealing approach and requires much less computational complexity than simulated annealing, to a problem of global energy minimization under a region constraint for object boundary extraction. In the method, with a given contour energy function, different target boundaries can be modeled as constrained global optimal solutions under different constraints expressed as a set of parameters characterizing the target 
contour interior structure. To search for the constrained global optimal solutions, the method takes a Lagrange approach and then introduces a quadratic constraint based on the square error of the current parameter values and the optimal values to convexify the Lagrange multiplier function [29]. The proposed method considers the deformable contour and the associated variables as random functions or variables in the convexified Lagrange multiplier; and then incorporates variational based deformable contour searching into MFA framework using saddle point approximation and combines it with an MFA parameter optimization scheme. High computational complexity is thus avoided. Notice that the mean field approximation used in [17] is not the same as the mean field annealing used in this formulation to achieve a global optimal solution.

In Section 2, related works on deformable contour methods are reviewed. In Section 3 and 4, MFA theory and the problem formulation are introduced and discussed. Section 5 details the derivation of the proposed approach. Illustrative examples and parameter setting experiments are shown in Section 6 and Section 7 respectively, while applications on biomedical images are demonstrated in Section 8. In section 9, the conclusion regarding this method is provided.

\section{Related works}

Deformable contour methods (DCMs), since originated by Kass et al [2], receive tremendous amount of attentions, and many efforts have been made to apply them to all sorts of problems. Typically, most DCMs [2] [4] [12] [13] model boundary extractions as energy minimization problems. Traditionally these methods often use functions of image gradient [2] [4] as contour energies. In cases where image gradient information is either noisy or inaccurate in identifying target boundary, the image gradient based energy functions often have multiple energy minima and as a result, these methods often result in undesired local energy minimum contour instead of target boundary. To deal with this problem, improvements in DCMs can be divided into two categories. 
In the first category, the methods [1] [5] [6] [7] [8] [16] propose new energy functions integrating region based image features to alleviate the problem of multiple energy minima. In [6] [7], region based energy functions firstly introduced in image classification methods are used to derive the deformable contour formulations and in [8] theses functions are further combined with the image gradient based contour energy functions to improve the performances. In general, the above methods are rather robust to noises and the segmentation is efficiency is improved [16]. However, in more challenging situations, in which a combination of region and image gradient information still can not accurately identify the target boundary (such as gaps and inhomogeneous interiors all present in one contour extraction problem), the methods still often result in undesired local energy minima; for instance, the resulting contours may either expand beyond the gaps or stops at noisy interiors. One way to handle the problem is to integrate priori shape model into the frameworks [18] [19] [20] to assist identifying target boundary. The problem with these methods is that a reliable shape model construction often requires intensive manual interventions.

The second category of DCMs model target boundary as global energy minimum [14] [15] and take global optimization approaches, mostly simulated annealing, to locate them. These methods are robust to undesired local energy minima. However, in a complex image context, with a snake based contour energy function, the target boundary is usually a global energy minimum under certain constraints (for instance, the constraints of the contour interior characteristics) instead of the actual global energy minimum contour throughout the entire image. Thus in [14] [15], either a major modification of the energy function, incorporating specific prior knowledge on target boundary [14] to make it be the global energy minimum contour, or a preset mask [15] constraining the contour searching space within a neighborhood close to target boundary is often required.

\section{A Brief Review of Mean Field Annealing}


Mean field annealing is a global optimization method derived from statistical mechanics [9]. Let $f$ be a random variable and $E(f)$ be an energy function to be minimized. Without any prior knowledge, the probability distribution of $f$ is assumed to be Gibbs distribution,

$$
P(f)=\frac{1}{Z} \exp \left(-\frac{1}{T} E(f)\right)
$$

with the partition function $Z$ being

$$
Z=\sum_{f \in P^{*}} \exp \left(-\frac{1}{T} E(f)\right)
$$

where $P^{*}$ is all the possible configurations of $f$ and $T$ is the temperature. The statistical mean of $f$ at temperature $T$ is defined as,

$$
\overline{f(T)}=\sum_{f \in P^{*}} f P(f)=\sum_{f \in P^{*}} f \frac{1}{Z} \exp \left(-\frac{1}{T} E(f)\right)
$$

According to mean field annealing theory [9], $\overline{f(T)}$ is of importance due to the well known fact that as the temperature approaches zero, $\overline{f(T)}$ approaches the global optimal point $f^{*}$,

$$
\lim _{T \rightarrow 0} \overline{f(T)}=\lim _{T \rightarrow 0} \sum_{f \in P^{*}} f P(f)=f^{*}
$$

This suggests that instead of minimizing $E(f)$ directly, we can try to evaluate mean field $\overline{f(T)}$ and then track $\overline{f(T)}$ from a sufficiently high temperature down to zero. The value of $\lim _{T \rightarrow 0} \overline{f(T)}$ is then the global optimal point.

In applications, $\overline{f(T)}$ can be evaluated using saddle point approximation theorem [9]. The theorem states that at temperature $T$, the partition function $Z$ can be approximated by

$$
Z \approx M \exp \left(-\frac{1}{T} E(\overline{f(T)})\right)
$$

where $M$ is a constant and $E(\overline{f(T)})$ satisfies $\left.\frac{\partial E(f)}{\partial f}\right|_{f=\overline{f(T)}}=0$. 
Note that in many applications, $f$ is a random function of time $t$ rather than a random variable. In these cases, the mean of $f$ at temperature $T$ is also a function of $t$.

\section{Problem Formulation}

\section{Problem statement}

Let $\Phi$ be an open domain subset of $\mathfrak{R}^{2}$ and $I(x, y): \Phi \rightarrow \Re$ be the image intensity function. Consider a target object with boundary $\Gamma(q)$ and interior $\Omega_{\Gamma}$ in the image. The average image intensity in $\Omega_{\Gamma}, I_{0}$, and the variance of image intensity in $\Omega_{\Gamma}, \sigma^{2}$, can be determined as

$$
I_{0}=\frac{\iint_{\Omega_{\Gamma}} I(x, y) d x d y}{\iint_{\Omega_{\Gamma}} d x d y},
$$

and

$$
\sigma^{2}=\frac{\iint_{\Omega_{\Gamma}}\left(I(x, y)-I_{0}\right)^{2} d x d y}{\iint_{\Omega_{\Gamma}} d x d y},
$$

We can model target boundary $\Gamma(q)$ as a close contour having the global minimum energy,

$$
E_{C}(\Gamma(q))=\oint_{\Gamma(q)} g(\nabla I(\Gamma(q)) \mid) d s
$$

satisfying

$$
D(x, y) \geq T_{V} \quad \text { if }(x, y) \in \Omega_{\Gamma}
$$

where $s$ is the normalized arc length, $q$ is the contour parameter. $g(|\nabla I(\Gamma(q))|)$ is a contour energy function firstly introduced in [4], which can be any positive decreasing function. In the paper, we choose

$$
g(|\nabla I(\Gamma(q))|)=\frac{1}{1+|\nabla I(\Gamma(q))|^{2}}+\alpha,
$$

$\nabla I(\Gamma(q))$ is the gradient of $I(x, y)$ with $(x, y)$ on $\Gamma(q), \alpha>0$ is a constant. As we can see from Eq. (7), when $\nabla I(\Gamma(q))$ is large, $g(|\nabla I(\Gamma(q))|)$ approaches its minimum $\alpha$; when $\nabla I(\Gamma(q))$ approaches zero, $g(|\nabla I(\Gamma(q))|)$ approaches its maximum $\alpha+1 . \alpha$ is related to contour smoothness. A large $\alpha$ indicates a smooth contour while a small $\alpha$ indicates just the opposite. 
$D(x, y)$, which is used to characterize $\Omega_{\Gamma}$, is a function of $I_{0}$ and $\sigma^{2}$. In the paper, we choose

$$
D(x, y)=\exp \left[-\frac{\left(I(x, y)-I_{0}\right)^{2}}{\beta \sigma^{2}}\right],
$$

where $\beta$ is a positive constant set as 2.0. $T_{V}>0$ is a threshold that can be adjusted for modeling different target boundaries as constrained global energy minima. A large $T_{V}$ often indicates a small admissible set of contours that satisfy the constraint of Eq. (6) while a small $T_{V}$ indicates just the opposite.

Then our problem is to estimate the optimal values of $\sigma^{2}, I_{0}$ and then find a close contour $C(q, t)$ enclosing region $\Omega_{c}(t)$ at time $t$, such that

$$
E_{C}(C(q, t))=\oint_{C} g(|\nabla I(C(q, t))|) d s
$$

is the global minimum under the constraint,

$$
D(x, y) \geq T_{V} \quad \text { for }(x, y) \in \Omega_{C}(t)
$$

The Lagrange formulation of Eq. (9) is

$$
L\left(C(q, t), \sigma^{2}, I_{0}\right)=\oint_{C} g(\nabla I(C(q)) \mid) d s-\lambda_{1} \iint_{\Omega_{C}}\left(D(x, y)-T_{V}\right) d x d y
$$

where $\lambda_{1}>0$ is a Lagrange multiplier. Since $\Gamma(q)$ is the constrained global energy minimum, according to Lagrange multiplier condition, the optimal setting of $\lambda_{1}, \lambda_{1}^{*}$, should satisfy

$$
\left.\frac{\partial L\left(C(q, t), \sigma^{2}, I_{0}, \lambda_{1}\right)}{\partial C(q, t)}\right|_{\substack{C(q, t)=\Gamma(q) \\ \lambda_{1}=\lambda_{1}^{*}}}=0
$$

According to [1],

$\frac{\partial L\left(C(q, t), \sigma^{2}, I_{0}, \lambda_{1}\right)}{\partial C(q, t)} \propto F\left(C(q, t), \sigma^{2}, I_{0}, \lambda_{1}\right)=\lambda_{1}\left[D(x(q, t), y(q, t))-T_{V}\right]+k g(|\nabla I(C(q, t))|)-\nabla g(|\nabla I(C(q, t))|) \cdot \vec{N}$

where $k$ is contour curvature and $\vec{N}$ is the normal direction of $C(q, t)$. Thus satisfying Eq. (12) is equivalent to satisfying $\quad F\left(\Gamma(q), \sigma^{2}, I_{0}, \lambda_{1}^{*}\right)=0$ 
In implementations, it may be difficult to find a $\lambda_{1}^{*}$ satisfying Eq. (13). As an alternative, we search for a $\lambda_{1}^{*}$ such that

$$
\left\|F\left(\Gamma(q), \sigma^{2}, I_{0}, \lambda_{1}^{*}\right)\right\|_{\mu}=\min \left\|F\left(\Gamma(q), \sigma^{2}, I_{0}, \lambda_{1}\right)\right\|_{\mu} \quad \text { for } \lambda_{1} \in \Re
$$

where $\|a\|_{\mu}$ is the $\mu$ norm function of $a$ and $\mu>0$. To simplify the computation of Eq. (14), we often choose $\mu$ as $\infty$, and $\lambda_{1}^{*}$ can then be determined. With this, Eq. (11) can be finally written as

$$
L\left(C(q, t), \sigma^{2}, I_{0}, \lambda_{1}^{*}\right)=\oint_{C} g(|\nabla I(C(q, t))|) d s-\lambda_{1}^{*} \iint_{\Omega_{C}}\left(D(x, y)-T_{V}\right) d x d y
$$

\section{A brief review of the original constrained optimization approach [1] [16] and its limitations}

To minimize Eq. (15), the original constrained optimization deformable contour method (CODCM) [1] initially estimates $\sigma^{2}, I_{0}, \lambda_{1}^{*}$ and then minimizes $L\left(C(q, t), \sigma^{2}, I_{0}, \lambda_{1}^{*}\right)$ using a variational approach. The contour evolution formula is

$$
\frac{\partial C(q, t)}{\partial t}=\left\{\lambda_{1}^{*}\left[D(x, y)-T_{V}\right]+k g(|\nabla I|)-\nabla g \cdot \vec{N}\right\} \vec{N}
$$

Since $\oint_{C} g\left(\nabla I(C(q, t) \mid) d s\right.$ is nonconvex having multiple local energy minima and $\lambda_{1}^{*}$ is often small, Eq.

(16) tends to be trapped into local energy minima. An extra constant balloon force $b$ has to be added,

$$
\frac{\partial C(q, t)}{\partial t}=\left\{\left(\lambda_{1}^{*}+b\right)\left[D(x, y)-T_{V}\right]+k g(|\nabla I|)-\nabla g \cdot \vec{N}\right\} \vec{N}
$$

The added balloon force $b$ can be viewed as to increase the weight of the region constraint. The difficulty with Eq. (17) is that with a small $b, L\left(C(q, t), \sigma^{2}, I_{0}, \lambda_{1}^{*}\right)$ is still nonconvex and has multiple minima while with a large $b$, locating the global minimum of $L\left(C(q, t), \sigma^{2}, I_{0}, \lambda_{1}^{*}\right)$ is equivalent to locating a local energy minimum near the maximum of $\iint_{\Omega_{C}}\left(D(x, y)-T_{V}\right) d x d y$, which is not necessarily the constrained global energy minimum. Though CODCM is rather successful in many applications [16], in more challenging situations, in which gaps and inhomogeneous interiors are all present in a single contour 
extraction problem, the methods may still either expand beyond the gaps (with a large $b$ ) or stops at the noisy interiors (with a small $b$ ).

Another problem is that when some a priori information about the target object is unavailable, an accurate estimation of $\sigma^{2}, I_{0}$, and $\lambda_{1}^{*}$ is often difficult especially when the object interior is inhomogeneous as shown in Fig. 6.1a; In [1], $I_{0}$ is computed as the mean brightness of the deforming contour and is updated during the contour deformation. Since $\lambda_{1}^{*}$ is small, $\lambda_{1}^{*}+b$ can be approximated by the constant $b . \sigma^{2}$ is the only parameter needs tuning.

\section{The Derivation of the Approach}

\section{Energy function formulation}

To overcome the difficulties of CODCM [1] [16], we add an extra constraint $E_{p}-T_{p} \leq 0$ that can convexify Eq. (15) without deviating the global optimal solutions,

where

$$
E_{p}=\left(\sigma^{2}-\sigma_{C}^{2}\right)^{2}+\left(I_{0}-I_{0}^{C}\right)^{2}+\left(\lambda_{1}^{*}-\lambda_{1}^{C}\right)^{2}
$$

$T_{p} \geq 0$ is a small constant. $\sigma_{C}^{2}, I_{0}^{C}$, and $\lambda_{1}^{C}$ are obtained from $\Omega_{C}(t)$ as follows:

$$
\begin{gathered}
I_{0}^{C}=\frac{\iint_{\Omega_{C}} I(x, y) d x d y}{\iint_{\Omega_{C}} d x d y}, \\
\sigma_{C}^{2}=\frac{\iint_{\Omega_{C}}\left[I(x, y)-I_{0}\right]^{2} d x d y}{\iint_{\Omega_{C}} d x d y}, \\
\left\|F\left(C(q, t), \sigma^{2}, I_{0}, \lambda_{1}^{C}\right)\right\|_{\mu}=\min \left\|F\left(C(q, t), \sigma^{2}, I_{0}, \lambda_{1}\right)\right\|_{\mu}
\end{gathered}
$$

Then Eq. (15) can be written as,

$$
L_{p}\left(C(q, t), \sigma^{2}, I_{0}, \lambda_{1}^{*}\right)=\overbrace{\oint_{C} g(\nabla I(C(q)) \mid) d s-\lambda_{1}^{*} \iint_{\Omega_{C}}\left(D(x, y)-T_{V}\right) d x d y}^{E_{I}}+\overbrace{\lambda_{2}\left(E_{p}-T_{p}\right)}^{E_{I}}
$$


where $\lambda_{2}>0$ is another Lagrange multiplier. $E_{I I}$ is a convex quadratic function [29] with the global minimum at $\Gamma(q)$. With the introduction of $E_{I I}$ and a large $\lambda_{2} \gg \lambda_{1}^{*}, L_{p}\left(C(q, t), \sigma^{2}, I_{0}, \lambda_{1}^{*}\right)$ is convexified with the global minimum at target boundary $\Gamma(q)$. The introduction of $E_{I I}$ is to require that the associated parameters of the resulting contour closed to those of target boundary $\Gamma(q)$. We can take a derivative based approach to minimize Eq. (22), which produces alternating procedures of contour evolution (computed according to Eq. (16)) and the updating of $\sigma^{2}, I_{0}$, and $\lambda_{1}^{*}$ (by letting $\sigma^{2}=\sigma_{C}^{2}$, $I_{0}=I_{0}^{C}$, and $\left.\lambda_{1}^{*}=\lambda_{1}^{C}\right)$. The problem is that the approach is a local method and often fails to locate global energy minimum.

\section{The derivations of the proposed approach}

To find the global optimal solution, we apply the framework of MFA discussed in Section 3 to minimize Eq. (22). Consider $C(q, t)$ as a random function of time $t$ and parameters $\sigma^{2}, I_{0}$, and $\lambda_{1}^{*}$ as random variables. Consider a proper temperature sequence $T_{i}, 1 \leq i \leq n$, satisfying $T_{i+1}<T_{i}, T_{n}=0$. According to MFA approach, $C(q, t), \sigma^{2}, I_{0}$, and $\lambda_{1}^{*}$ can be regarded as all random functions of temperature $T_{i}$. Thus we denote $C(q, t), \sigma^{2}, I_{0}$, and $\lambda_{1}^{*}$ as $C\left(q, t, T_{i}\right), \sigma^{2}\left(T_{i}\right), I_{0}\left(T_{i}\right), \lambda_{1}^{*}\left(T_{i}\right)$. Our strategy to search for the global optimum of $C(q, t), \sigma^{2}, I_{0}$, and $\lambda_{1}^{*}$ is to track the mean values of $C\left(q, t, T_{i}\right), \sigma^{2}\left(T_{i}\right), I_{0}\left(T_{i}\right)$, and $\lambda_{1}^{*}\left(T_{i}\right)$ until $T_{i}$ drops to zero as $i$ goes from 1 to $n$. To estimate the mean values of $C\left(q, t, T_{i}\right), \sigma^{2}\left(T_{i}\right)$, $I_{0}\left(T_{i}\right)$, and $\lambda_{1}^{*}\left(T_{i}\right)$, according to [9], we can ignore the correlations of the mean field of $C\left(q, t, T_{i}\right)$, $\sigma^{2}\left(T_{i}\right), I_{0}\left(T_{i}\right), \lambda_{1}^{*}\left(T_{i}\right)$ and proceed to update each variables separately by holding all other parameters unchanged. Let $C\left(q, 0, T_{1}\right)$ be the given initial contour denoted as $C\left(T_{1}\right)$. Let $\sigma_{i n}^{2}\left(T_{1}\right)=\sigma_{C\left(T_{1}\right)}^{2}$, $I_{0}^{i n}\left(T_{1}\right)=I_{0}^{C\left(T_{1}\right)}$, and $\lambda_{1}^{\text {in }}\left(T_{1}\right)=\lambda_{1}^{C\left(T_{1}\right)}$. Let $i=1$ and go to Step 1 .

Step1 is to keep $\sigma^{2}\left(T_{i}\right)=\sigma_{i n}^{2}\left(T_{i}\right), I_{0}\left(T_{i}\right)=I_{0}^{i n}\left(T_{i}\right), \lambda_{1}^{*}\left(T_{i}\right)=\lambda_{1}^{i n}\left(T_{i}\right)$ constant and compute the mean value of $C\left(q, t, T_{i}\right)$. By MFA theory discussed in Section 3, the mean of $C\left(q, t, T_{i}\right)$ can be evaluated from 
partition function using the saddle point approximation. At temperature $T_{i}$, the partition function $Z$ can be approximated by

$$
Z \approx M \exp \left(-\frac{1}{T_{i}} L_{p}\left(\overline{C\left(q, t, T_{i}\right)}, \sigma^{2}\left(T_{i}\right), I_{0}\left(T_{i}\right), \lambda_{1}^{*}\left(T_{i}\right)\right)\right)
$$

where $\left.\frac{\partial L_{p}\left(C(q, t), \sigma^{2}\left(T_{i}\right), I_{0}\left(T_{i}\right), \lambda_{1}^{*}\left(T_{i}\right)\right)}{\partial C(q, t)}\right|_{C(q, t)=\overline{C\left(q, t, T_{i}\right)}}=0 . \quad$ In applications, the variations of $\lambda_{1}^{C}, \sigma_{C}^{2}$, and $I_{0}^{C}$ are much slower comparing to that of $C(q, t)$ therefore we neglect their fluctuations during the deformation of $C(q, t)$. With this, according to [1], $\overline{C\left(q, t, T_{i}\right)}$ can then be computed by letting,

$$
\left.\frac{\partial C(q, t)}{\partial t}\right|_{\substack{C(q, t)=\overline{C\left(q, t T_{i}\right)} \\ C(q, 0)=C\left(T_{i}\right)}}=\left.\frac{\partial L_{p}\left(C(q, t), \sigma^{2}\left(T_{i}\right), I_{0}\left(T_{i}\right), \lambda_{1}^{*}\left(T_{i}\right)\right)}{\partial C(q, t)}\right|_{C(q, t)=\overline{C\left(q, t T_{i}\right)}}=0
$$

where

$$
\frac{\partial C(q, t)}{\partial t}=\left\{\lambda_{1}^{*}\left(T_{i}\right)\left[D\left(x, y, T_{i}\right)-T_{V}\right]+k g(\nabla I \mid)-\nabla g \cdot \vec{N}\right\} \vec{N}
$$

and

$$
D\left(x, y, T_{i}\right)=\frac{\left(I(x, y)-I_{0}\left(T_{i}\right)\right)^{2}}{\beta \sigma^{2}\left(T_{i}\right)} .
$$

To implement constraint of Eq. (23), we relax it as

$$
\left.\frac{\partial C(q, t)}{\partial t}\right|_{\substack{\left.C(q, t)=\overline{C(q, t} T_{i}\right) \\ C(q, 0)=C\left(T_{i}\right)}} \leq T_{C}
$$

where $T_{C} \geq 0$ is a given constant. To reduce computational complexity, instead of satisfying Eq. (23), contour $C(q, t)$ is deformed for a certain time interval $l>0$ and we assume that Eq. (25) can be satisfied after the interval. Then $\overline{C\left(q, t, T_{i}\right)}$ can be computed according to Eq. (24) by letting $\overline{C\left(q, t, T_{i}\right)}=C(q, l)$ with $C(q, 0)=C\left(T_{i}\right)$ being the initial contour.

Step2 is to keep $\overline{C\left(q, t, T_{i}\right)}$ constant and calculate $\sigma^{2}\left(T_{i}\right), I_{0}\left(T_{i}\right), \lambda_{1}^{*}\left(T_{i}\right)$. We denote the mean values of $\sigma^{2}\left(T_{i}\right), I_{0}\left(T_{i}\right), \lambda_{1}^{*}\left(T_{i}\right)$ as $\overline{\sigma^{2}\left(T_{i}\right)}, \overline{I_{0}\left(T_{i}\right)}, \overline{\lambda_{1}^{*}\left(T_{i}\right)}$ and $\sigma_{C}^{2}\left(T_{i}\right), I_{0}^{C}\left(T_{i}\right), \lambda_{1}^{C}\left(T_{i}\right)$ as values of $\sigma_{C}^{2}, I_{0}^{C}$, and $\lambda_{1}^{C}$ computed from $\overline{C\left(q, t, T_{i}\right)}$, respectively. With the derivation given in the Appendix, we have

$$
\overline{I_{0}\left(T_{i}\right)}=I_{0}^{C}\left(T_{i}\right),
$$




$$
\overline{\sigma^{2}\left(T_{i}\right)}=\sigma_{C}^{2}\left(T_{i}\right)+\sqrt{\frac{T_{i}}{\pi}}
$$

and

$$
\overline{\lambda_{1}^{*}\left(T_{i}\right)}=\lambda_{1}^{C}\left(T_{i}\right)+\sqrt{\frac{T_{i}}{\pi}}
$$

Then let

$$
I_{0}^{i n}\left(T_{i+1}\right)=\overline{I_{0}\left(T_{i}\right)}, \sigma_{i n}^{2}\left(T_{i+1}\right)=\overline{\sigma^{2}\left(T_{i}\right)}, \lambda_{1}^{i n}\left(T_{i+1}\right)=\overline{\lambda_{1}^{*}\left(T_{i}\right)}, C\left(T_{i+1}\right)=\overline{C\left(q, t, T_{i}\right)} .
$$

Let $i=i+1$ and go to Step1 until temperature drops to zero.

\section{Comments:}

The above solution includes two alternative procedures of contour deformation of Eq. (24) and parameter updating of Eq. (26) to (28). Eq. (24) is similar to Eq. (16) of CODCM [1]. However, in Eq. (24), the values of $\sigma^{2}\left(T_{i}\right), I_{0}\left(T_{i}\right)$, and $\lambda_{1}^{*}\left(T_{i}\right)$ vary during the contour deformation as shown in Eq. (26) to Eq. (28). From Eq. (27), it is easy to see that $\sigma^{2}\left(T_{i}\right)$ is large when temperature is high and $\sigma^{2}\left(T_{i}\right)$ gradually reduces when the temperature lowers as the contour grow outward. The value of temperature $T_{i}$ controls the dynamic range of $\sigma^{2}\left(T_{i}\right)$ thus determines the method's adaptability to the inhomogeneous interiors and noises. It is also interesting to note that according to Eq. (28) the magnitude of the balloon force velocity term in Eq. (24) is decaying during the contour evolution process. This feature provides the method more robustness to noisy interiors while enhancing its performances in situations of gaps and blur boundaries. In general, the whole contour deformation process can be viewed as an annealing process, in which contour flows outward in a high temperature and then cools down and anneals near the target boundary.

\section{Algorithm description}

From the above discussions, an iterative algorithm can be derived as follows:

For an initial contour $C(q, t)$ with the size of 5 by $5, t=0$ and interior $\Omega_{C}(t)$, at temperature $T_{i}=T_{\text {init }}$ $i=1$, where $T_{\text {init }}$ is the initial temperature, let $\overline{C\left(q, t, T_{i}\right)}=C(q, t)$ do

i) Compute $\sigma_{C}^{2}, I_{0}^{C}$, and $\lambda_{1}^{C}$ from $\overline{C\left(q, t, T_{i}\right)}$ using Eq. (19), Eq. (20), and Eq. (21). 
ii) Update $\overline{\sigma^{2}\left(T_{i}\right)}, \overline{I_{0}\left(T_{i}\right)}$, and $\overline{\lambda_{1}^{*}\left(T_{i}\right)}$ using Eq. (26), Eq. (27), and Eq. (28).

iii) Let $T_{i+1}=T_{i}^{*} \operatorname{dec} T$, and update $\sigma_{i n}^{2}\left(T_{i}\right), I_{0}^{i n}\left(T_{i}\right), \lambda_{1}^{i n}\left(T_{i}\right)$, and $C\left(T_{i}\right)$ according to Eq. (29), where $0<\operatorname{dec} T<1$ is the updating factor.

iv) Evolve $C(q, t)$ for $l$ iterations ( $l>0$ is a constant) according to Eq. (24) using narrow band numerical scheme [3] with $C(q, 0)=C\left(T_{i}\right)$ being the initial contour. Let $\overline{C\left(q, t, T_{i}\right)}=C(q, l)$. Stop when the maximum velocity of $C(q, l)$ is smaller than a threshold $V_{t}$ or a maximum iteration number $t_{m}$ has been reached, where $V_{t}$ and $t_{m}$ are positive constants. To further increase the robustness of the algorithm to the setting of iteration number $t_{m}$, we compute the value of contour energy according to Eq. (9) and record the lowest energy contour. The output contour is then the contour with lowest contour energy.

It should be noted that we can also set the stopping criteria as when the temperature $T_{i}$ drops to zero. However, this stopping criterion is too sensitive to the settings of $T_{\text {init }}$ and $\operatorname{dec} T$ thus is not used.

\section{Some remarks on Appendix:}

As illustrated in Appendix, if $\overline{\Omega_{C}\left(t, T_{1}\right)} \subseteq \Omega_{\Gamma}$, where $\overline{\Omega_{C}\left(t, T_{1}\right)}$ is the interior of $\overline{C\left(q, t, T_{1}\right)}$ and $\Omega_{\Gamma}$ is the interior of target boundary, there exists at least one temperature sequence $T_{i}, 1 \leq i \leq n$ such that $\overline{C\left(q, t, T_{i}\right)}$ computed according to Eq. (24) and Eq. (26) to (28) satisfies $\overline{\Omega_{C}\left(t, T_{i}\right)} \subseteq \Omega_{\Gamma}$. In applications, we assume that $\overline{\Omega_{C}\left(t, T_{i}\right)} \subseteq \Omega_{\Gamma}$ can be satisfied by a careful selection of $T_{\text {init }}$ and $\operatorname{dec} T$. Tuning $T_{i n i t}$ and $\operatorname{dec} T$ are thus needed in some applications. As an advantage, with the satisfaction of $\overline{\Omega_{C}\left(t, T_{i}\right)} \subseteq \Omega_{\Gamma}$, the method can be applied to extract any target boundary $\Gamma(q)$ that can be modeled as the global energy minimum contour with the constraint $\Omega_{C}^{i n} \subseteq \Omega_{\Gamma}$, where $\Omega_{C}^{i n}$ is the interior of an arbitrary contour inside $\Omega_{\Gamma}$, as shown in Fig. 8.4 and 8.5. In the viewpoint of global optimization, the physical meaning of the solutions Eq. (24) and (26) to (28) is that since $\overline{\Omega_{C}\left(t, T_{i}\right)} \subseteq \Omega_{\Gamma}$ is satisfied and the contour is mainly moving 
outward, when $i$ approaches $n, \overline{C\left(q, t, T_{i}\right)}$ is more closed to the target boundary and $\sigma_{C}^{2}\left(T_{i}\right), I_{0}^{C}\left(T_{i}\right)$, $\lambda_{1}^{C}\left(T_{i}\right)$ are more close to $\sigma^{2}, I_{0}$, and $\lambda_{1}^{*}$.

\section{Illustrative Examples}

In this section, we will show that different target boundaries in a single image can be modeled as the constrained global minima with different settings of $T_{V}$. We then illustrate the processes of locating these constrained global energy minima using the proposed method with a comparison to the results using Eq. (17) of CODCM [1] assuming that $\sigma^{2}$ and $I_{0}$ of the target objects are known.

The examples shown in Fig. 6.1(a) is a 165 by 165 image designed with three overlapping circles $M C_{1}$, $M C_{2}, M C_{3}$ of size $r_{1}=40$ pixels $\left(M C_{1}\right), r_{2}=55$ pixels $\left(M C_{2}\right), r_{3}=70$ pixels $\left(M C_{3}\right)$ at different center locations, where $r_{i}, i=1,2,3$ is the radius of circle $M C_{i}$. The interior brightness of circles $M C_{1}$ and $M C_{3}$ is radially decreasing from the centers to their perimeters in a straight line fashion for circle $M C_{1}$ from 255 to 207 , circle $M C_{3}$ from 160 to 69 with the scale of 255 gray levels while the interior brightness of $M C_{2}$ is radially increasing from the center to the perimeter in a straight line fashion from 40 to 150 . The values of $\sigma^{2}, I_{0}$, and $D_{\min }(x, y)$ inside $M C_{1}, M C_{2}, M C_{3}$, and the respective contour energy $E_{C}$ (computed according to Eq. (9)) are listed in the Table 6.1, where $D_{\min }(x, y)$ is the minimum of $D(x, y)$,

\begin{tabular}{|c|c|c|c|c|}
\hline & $D_{\text {min }}(x, y)$ & $\sigma^{2}$ & $I_{0}$ & $E_{C}$ \\
\hline$M C_{1}$ & 0.016 & 127.7 & 223 & 0.00383 \\
\hline$M C_{2}$ & 0.289 & 2359 & 178 & 0.00547 \\
\hline$M C_{3}$ & 0.174 & 3682 & 142 & 0.00411 \\
\hline
\end{tabular}

Table 6.1 The parameters and contour energy for $M C_{1}, M C_{2}$, and $M C_{3}$

It is easy to see that when $T_{V}$ is set smaller than 0.016 , the value of $D_{\min }(x, y)$ inside $M C_{1}, M C_{1}$ is the constrained global energy minimum. When $T_{V}$ is larger than 0.016 but smaller than $D_{\text {min }}(x, y)$ inside $M C_{3}, 0.174$, the constrained global energy minimum is $M C_{3}$. When $T_{V}$ is larger than 0.174 but smaller than $D_{\text {min }}(x, y)$ inside $M C_{2}, 0.289, M C_{2}$ becomes the constrained global energy minimum. Thus $M C_{1}$, 
$M C_{2}, M C_{3}$ can all be modeled as the constrained global energy minimum by setting different values of $T_{V}$, respectively $T_{V}=0.01$ for $M C_{1}, T_{V}=0.1$ for $M C_{3}, T_{V}=0.25$ for $M C_{3}$. The extraction processes of $M C_{1}, M C_{2}, M C_{3}$ are shown in Fig. 6.1b to 6.1e, 6.2a to 6.2e, and 6.3a to 6.3e. With the same initial contour position indicated as the black dot in Fig. 6.1a, the method successfully locates all the constrained global energy minimum contours. In the experiments, we set $\operatorname{dec} T$ as 0.966 and respectively set $T_{\text {init }}=10^{6}$ for $M C_{1}, T_{\text {init }}=10^{8}$ for $M C_{2}$, and $T_{\text {init }}=10^{11}$ for $M C_{3}$. The results of [1] using the same initial contour in Fig. 6.1a and Eq. (17) by assuming that $I_{0}$ and $\sigma^{2}$ of $M C_{1}, M C_{2}$, and $M C_{3}$ are known (as listed in Table 6.1) are shown in Fig. 6.4b to 4e, 5a to 5e, and 6a to 6e. Though Eq. (17) successfully extracts $M C_{1}$, it fails to extract $M C_{2}$ and $M C_{3}$. To illustrate the key differences between the behaviors of the two methods, Fig. 6.7a to 6.7e show the scaled images of $D\left(x, y, T_{i}\right)-T_{V}=\frac{\left(I(x, y)-I_{0}\left(T_{i}\right)\right)^{2}}{\beta \sigma^{2}\left(T_{i}\right)}-T_{V}$ in Eq. (24) under the temperatures respectively corresponding to Fig. 6.3a to 6.3e; and Fig. 6.8a show the scaled image of $D(x, y)-T_{V}=\frac{\left(I(x, y)-I_{0}^{M C_{3}}\right)^{2}}{\beta \sigma_{M C_{3}}^{2}}$ in Eq. (17), where $I_{0}^{M C_{3}}$ and $\sigma_{M C_{3}}^{2}$ are the mean brightness and variance of image intensity inside $M C_{3}$. We note that $\mathrm{n}$ Fig. 6.7a and $6.7 \mathrm{~b}$ the variations of $D\left(x, y, T_{i}\right)-T_{V}$ are small and the fluctuations of the brightness distributions across $M C_{1}, M C_{2}$, and $M C_{3}$ are only mildly reflected in contour velocity. Therefore it is easy for the contour to overcome the inhomogeneous interiors of $M C_{3}$. From 6.7c to 6.7e, the variations of $D\left(x, y, T_{i}\right)-T_{V}$ become larger and the steep fluctuations of the brightness distributions across $M C_{1}, M C_{2}, M C_{3}$ becomes more evidently reflected in contour velocity. Noting that the contour has already marched over most sections of $M C_{1}$, $M C_{2}$ and has been closed to $M C_{3}$ as shown in Fig. 6.3c to 6.3e, the steep variations of $D\left(x, y, T_{i}\right)-T_{V}$ can only help the contour stop at target boundary $M C_{3}$. In Fig. 6.8a, the constant distribution of $D(x, y)-T_{V}$ using CODCM [1], though can stop the contour near $M C_{3}$, is inhomogeneous inside $M C_{3}$ and the contour has difficulties in marching over $M C_{1}$. Thus the method [1] fails to extract $M C_{3}$. 


\section{Parameter Setting Experiments}

The proposed algorithm has over all ten parameters: $T_{V}$ (Eq. (6)), $\alpha$ (Eq. (7)), $\beta$ (Eq. (8)), threshold $T_{p}$ (Eq. (22)), multipliers $\lambda_{2}$ (Eq. (22)), $l$ (iteration number per parameter updating in algorithm Step iv), velocity threshold $V_{t}$, maximum iteration number $t_{m}$, initial temperature $T_{i n i t}$, and temperature updating factor $\operatorname{dec} T$. In the method, we do not need to tune $T_{p}$ and $\lambda_{2}$ since their setting does not affect solution. Velocity threshold $V_{t}$ and maximum iteration number $t_{m}$ are common parameters for level set algorithms [3] and in all the experiments, we keep $V_{t}$ constant as 0.005 and $t_{m}$ as $120 . \beta$ is an implicit parameter of CODCM [1] and is set constant 2.0. $l$ is related to the time step of every level set iteration and is set constant as 1 . Thus in the method, there are four parameters, $T_{V}, \alpha, T_{\text {init }}$ and $\operatorname{dec} T$, need tuning. The setting of $\alpha$ is related to contour smoothness and is set large for boundaries with large gaps. In case of small or no large gap, $\alpha$ is often set as 0.01 .

In the following, we will illustrate the experiments that evaluate the effects using different settings of $T_{V}$, $T_{\text {init }}$, and $\operatorname{dec} T$ on energy minimization. To facilitate the evaluations, we keep $\alpha=0.01$ unchanged and assume that $\overline{\Omega_{C}\left(t, T_{i}\right)} \subseteq \Omega_{\Gamma}$ is satisfied as the contour evolves. The first experiment is to evaluate the effect of the setting of $T_{V}$ on constrained global energy minimization. We keep $T_{\text {init }}$ as $10^{7}$ and DecT as 0.9 unchanged, then reduce the values of $T_{V}$ and evaluate the energies of the resulting contours corresponding to different $T_{V}$ s. With smaller $T_{V}$, the region constraint is more relaxed and the resulting contours tend to have lower energy. Fig. 7.1a is a 256 by 256 synthetic image. The image is composed of four concentric circles, $C R_{i}, i=1 \sim 4$, with their brightness radially distributed according to sine function and edges sharpened by four edge profile functions. The values of $D_{\min }(x, y), \sigma^{2}, I_{0}$ inside each of the circles and the respective contour energy $E_{C}$ are listed in the following table, 


\begin{tabular}{|l|c|c|c|c|}
\hline & $D_{\text {min }}(x, y)$ & $\sigma^{2}$ & $I_{0}$ & $E_{C}$ \\
\hline$C R_{1}$ & 0.395 & 917 & 214 & 0.0173 \\
\hline$C R_{2}$ & 0.158 & 744 & 203 & 0.0157 \\
\hline$C R_{3}$ & 0.044 & 636 & 192 & 0.0111 \\
\hline$C R_{4}$ & 0.0087 & 570 & 182 & 0.0098 \\
\hline
\end{tabular}

Table 7.1 The parameters and energy for $C R_{1}, C R_{2}, C R_{3}$, and $C R_{4}$

In the experiment, the initial contour is set at the center of circles $C R_{i}, i=1 \sim 4$ and we respectively choose $T_{V}$ as $0.0001,0.03,0.1$, and 0.35 . The resulting contours $C R_{1}, C R_{2}, C R_{3}$, and $C R_{4}$ are shown in Fig. $7.1 \mathrm{~b}$ to 7.1e. From the experiment, we can see that with a small $T_{V}=0.0001, C R_{1}, C R_{2}, C R_{3}$, and $C R_{4}$ all satisfy the region constraint thus the contour with lowest energy is resulted. As the value of $T_{V}$ increases, fewer contours satisfy the region constraint thus contours with higher energy are resulted as shown in Table 7.1.

The second experiment is to evaluate the effect of the settings of $\operatorname{dec} T$ and $T_{\text {init }}$ on constrained global energy minimization. In the experiment, we keep $T_{V}=0.001$ unchanged. The experiments are conducted in a 256 by 256 MRI brain image shown in Fig. 7.2a with the initial location I marked as a white dot shown in Fig. 7.2b. As we can see, in Fig. 7.2a, the interior of the intracranial is very in homogeneous, where numerous local energy minima exist. Since $T_{V}=0.001$ is small, most of the local energy minimum contours satisfy the region constraint of Eq. (10). By evaluating the energies of the resulting contours with different $\operatorname{dec} T$ and $T_{\text {init }}$, we will show that for a given value of $\operatorname{dec} T$ (or $T_{\text {init }}$ ), a higher $T_{\text {init }}$ (or decT) is more capable of overcoming local energy minima and results lower contour energy while a lower $T_{\text {init }}$ (or $\operatorname{dec} T$ ) indicates just the opposite.

To test the effect of the settings of $T_{\text {init }}$, we set $\operatorname{dec} T=0.85$ and conduct the experiments by increasing the value of $T_{\text {init }}$ from $T_{\text {init }}=5^{*} 10^{5}$ to $T_{\text {init }}=5^{*} 10^{9}$. The energies of the resulting contours with different settings of $T_{\text {init }}$ are listed in Table 7.2. One can observe that increasing $T_{i n i t}$ has the effects of producing contours with lower contour energy. To test the effect of the settings of $\operatorname{dec} T$, we set $T_{\text {init }}=5^{*} 10^{6}$ and 
conduct the experiments by increasing the value of $\operatorname{dec} T$ from 0.85 to 0.98 with the energies of the resulting contours listed in Table 7.3. One can also observe that increasing $\operatorname{dec} T$ has the effects of increasing the capability of overcoming local contour energy minima and resulting lower energy contour. The third experiment is to test the sensitivity of the algorithm to the positions of the initial contour. In order to quantify the sensitivity, we pick two extra initial points, initial point II and III, besides initial point I shown in Fig 7.2b and compute the average distance between the resulting contours using these initial contours. We take two measures to quantify the distances between different contours. The first distance measure is introduced in [27], which is written as $\varepsilon_{1}=1-\frac{|T P \cap E P|}{|T P \cup E P|}$ with $T P$ denoting the set of pixels inside one of the resulting contour $C_{1}$ and $E P$ denoting the set of pixels inside another resulting contour $C_{2}$. The measure $\varepsilon_{1}$ is an indicator of the overall distance between the resulting contours. The second distance measure is $\varepsilon_{2}=\max _{X \in C_{1} Y \in C_{2}}^{\min } \operatorname{dist}(X, Y)$ with $\operatorname{dist}(X, Y)$ function representing the Euclidean distance between the integer coordinates of the pixels $X$ and $Y . \varepsilon_{2}$ is a local measure useful in determining the distance between the high curvature portions of the resulting contours. To test the sensitivity of $\varepsilon_{1}$ and $\varepsilon_{2}$ to $T_{i n i t}$, we keep $\operatorname{dec} T$ unchanged at 0.85 and increase $T_{\text {init }}$ from $5^{*} 10^{6}$ to $5 * 10^{8}$. To test the sensitivity to $\operatorname{dec} T$, we keep $T_{\text {init }}$ unchanged at $5 * 10^{6}$ and increase $\operatorname{dec} T$ from 0.85 to 0.98. The computed average distances are listed in Table 7.4. As we can see from the data, the algorithm becomes to be more sensitive when $T_{\text {init }}$ and $\operatorname{dec} T$ increase and vice versa.

Table 7.1

$$
\begin{array}{|c|c|c|c|c|c|}
\hline & T_{\text {init }}=5 * 10^{5} & T_{\text {init }}=5 * 10^{6} & T_{\text {init }}=5 * 10^{7} & T_{\text {init }}=5 * 10^{8} & T_{\text {init }}=5 * 10^{9} \\
\hline \text { Contour Energy } & 0.04144 & 0.030626 & 0.030192 & 0.024595 & 0.0185531 \\
\hline
\end{array}
$$

Table 7.2 


\begin{tabular}{|l|r|r|r|r|r|}
\hline & DecT=0.85 & DecT=0.92 & DecT=0.94 & DecT=0.96 & DecT=0.98 \\
\hline Contour Energy & 0.030626 & 0.0299965 & 0.0251968 & 0.0163892 & 0.0141091 \\
\hline
\end{tabular}

Table 7.3

\begin{tabular}{|c|c|c|}
\hline & $\varepsilon_{1}$ & $\varepsilon_{2}$ \\
\hline$T_{\text {init }}=5 * 10^{6}$, Dec $T=0.85$ & 0.1195 & 5.886 \\
\hline$T_{\text {init }}=5 * 10^{7}$, Dec $T=0.85$ & 0.1505 & 9.025 \\
\hline$T_{\text {init }}=5 * 10^{8}$, Dec $T=0.85$ & 0.1725 & 22.923 \\
\hline$T_{\text {init }}=5 * 10^{6}$, Dec $T=0.94$ & 0.1295 & 18.669 \\
\hline$T_{\text {init }}=5 * 10^{6}$, Dec $T=0.98$ & 0.134 & 20.695 \\
\hline
\end{tabular}

\section{Applications}

Our experimental evaluations and comparisons can be divided into three separate items and we separately discuss each category as follows:

i) Contour evaluations are performed on a set of challenging contour extraction problems including ultrasound pig heart images having noisy contour interiors, sharp contour segment protrusions, and gaps as shown in Fig. 8.1, MRI knee images having thick and very blur contour segment and contour-withincontour segment as shown in Fig. 82, and MRI brain images having contours with complex shape, inhomogeneous interiors, and blur segments as shown in Figs. 8.3, 8.4, and 8.5. All resulting contours are shown on the right-hand side of their originals in Figs. 8.1, 8.2, 8.4 and 8.5. Notice that for the MRI brain images, we perform three separate extractions of external boundary of intracranial (Fig. 8.3), cerebral boundary (Fig. 8.4), and sulci boundary (Fig. 8.5). Furthermore, Fig. 8.3 shows a sequence of contour progression images. All these contours are considered very good results. 
ii) A performance comparison between the proposed method and CODCM [16] is made on four images shown in Figs. 8.6 (a), 8.6(b), 8.7(a), and 8.8(a) of visual blood cell image, MRI knee image, and two MRI brain images respectively. In Fig. 8.6(a), the cells have rather large gaps and in Fig. 8.6(b), there are blur boundary segment and a rather inhomogeneous interior. In Fig. 8.7(a) and 8.8(a), there are very inhomogeneous interiors inside the external boundaries of intracranial. As we see from the results shown in Fig. 8.6(c) to 8.6(f), 8.7(b), 8.7(c), 8.8(b), 8.8(c), comparing to the result of [16], substantial improvements can be seen.

iii) The proposed method is also compared to other conventional deformable contour methods; the first

two methods are geodesic snake [4], and area \& length active contour [10] using $h(x, y)=\frac{1}{1+\left|\nabla G^{*} I\right|^{2}}$ as the edge detection function and the third method is T-snake [11]. We select two zoomed images of Fig. 8.9a a stomach CT image with additive noise of Gaussian noise (variance 3000), and Fig. 8.10a a midline sagittal MRI brain image. Similar to all other three methods, a Gaussian filter $N(0,1)$ is applied to both images as a preprocessing operation. No a priori information of object shape or brightness distribution is assumed (that is why [6][7][8] are not included). To provide an objective comparison, two sets of three dark dots in Fig. 8.9a and Fig. 8.10a are used as initial candidate locations for all four methods including ours. Each method using an initial candidate location provides a resulting contour. The best contour (best of the three resulting contours for Fig. 89a and Fig. 8.10a) of each method from all initial candidate locations is selected for comparison. Comparing these resulting contours, our proposed method has the best contours. It should be noted that similar comparisons have been conducted in [16] and a thorough comparison with quantitative analysis may be found in [28].

\section{Conclusions}

In this paper, a constrained global optimization formulation has been proposed for boundary extraction problems. The formulation overcomes the problems of multiple local energy minima while preserves good controllability in extracting target boundaries with different region characteristics. The 
effectiveness of the approach in locating constrained globalenergy minima is evaluated in a synthetic test image, where constrained global energy minima are known. The results are compared to those of CODCM [1] with the same energy function and region constraint, where an accurate estimation of the object interior features is assumed. The experiment proves that the contribution of the method can not be reduced as only providing a dynamic parameter estimation to avoid manual parameter tuning. The performance of the method is then demonstrated on very challenging segmentation applications and is compared to those of other deformable contour methods and substantial improvements in handling segmentation difficulties including gaps, noises, and blur boundaries are reported. The method is computationally efficient usually taking 10 seconds to 2 minutes on workstation Ultra Sun Blade 100 for most applications.

\section{Appendix}

Noting that in Eq. (22) $\lambda_{1}^{*}<<\lambda_{2}$ and $L_{p} \approx E_{I I}, \overline{I_{0}\left(T_{i}\right)}$ can be computed as

$$
\overline{I_{0}\left(T_{i}\right)}=\frac{\int_{-\infty}^{\infty} I_{0} e^{-L_{p}\left(I_{0}\right)} d I_{0}}{\int_{-\infty}^{\infty} e^{-L_{p}\left(I_{0}\right)} d I_{0}} \approx \frac{\int_{-\infty}^{\infty} I_{0} e^{-E_{I I}\left(I_{0}\right)} d I_{0}}{\int_{-\infty}^{\infty} e^{-E_{I I}\left(I_{0}\right)} d I_{0}}=I_{0}^{C}\left(T_{i}\right) .
$$

In the following, we compute $\sigma^{2}\left(T_{i}\right)$ and $\lambda_{1}^{*}\left(T_{i}\right)$. We assume that $\overline{\Omega_{C}\left(t, T_{1}\right)} \subseteq \Omega_{\Gamma}$, where $\overline{\Omega_{C}\left(t, T_{1}\right)}$ is the interior of $\overline{C\left(q, t, T_{1}\right)}$. Noting that $C\left(q, 0, T_{1}\right)$ is a 5 by 5 contour inside target boundary $\Gamma(q)$, the condition can be easily satisfied in most applications. Let $i=1 . \sigma^{2}\left(T_{i}\right)$ should satisfy

$$
\min D(x, y)=\min \exp \left(-\frac{\left(I(x, y)-I_{0}\right)^{2}}{\beta \sigma^{2}\left(T_{i}\right)}\right) \geq T_{V} \quad \text { for }(x, y) \in \overline{\Omega_{C}\left(t, T_{i}\right)}
$$

Let $\sigma_{o}^{2}$ satisfy $\quad \min \exp \left(-\frac{\left(I(x, y)-I_{0}\right)^{2}}{\beta \sigma_{o}^{2}}\right)=T_{V} \quad$ for $(x, y) \in \overline{\Omega_{C}\left(t, T_{i}\right)}$

It is obvious that $\sigma^{2}\left(T_{i}\right) \geq \sigma_{o}^{2}$. Since the computation of $\sigma^{2}\left(T_{i}\right)$ based on $\sigma_{o}^{2}$ is rather complex, in implementations, we approximate $\sigma_{o}^{2}$ by $\sigma_{C}^{2}\left(T_{i}\right)$ and compute $\sigma^{2}\left(T_{i}\right)$ as, 


$$
\overline{\sigma^{2}\left(T_{i}\right)}=\frac{\int_{\sigma_{C}^{2}}^{\infty} \sigma^{2} e^{-L_{p}\left(\sigma^{2}\right)} d \sigma^{2}}{\int_{\sigma_{C}^{2}}^{\infty} e^{-L_{p}\left(\sigma^{2}\right)} d \sigma^{2}} \approx \frac{\int_{\sigma_{C}^{2}}^{\infty} \sigma^{2} e^{-E_{I l}\left(\sigma^{2}\right)} d \sigma^{2}}{\int_{\sigma_{C}^{2}}^{\infty} e^{-E_{I I}\left(\sigma^{2}\right)} d \sigma^{2}}=\sigma_{C}^{2}\left(T_{i}\right)+\sqrt{\frac{T_{i}}{\pi}}
$$

Similarly, the estimated value of $\lambda_{1}^{*}\left(T_{i}\right)$ has to satisfy

$$
\min \left(\frac{\partial \overline{C\left(q, t, T_{i}\right)}}{\partial t}\right)=\min \left[\left\{\lambda_{1}^{*}\left(T_{i}\right)\left[D\left(x, y, T_{i}\right)-T_{V}\right]+k g(|\nabla I|)-\nabla g \cdot \vec{N}\right\} \vec{N}\right] \geq 0 \quad \text { for }(x, y) \in \overline{C\left(q, t, T_{i}\right)}
$$

Let $\lambda_{1}^{o}$ be $\quad \min \left[\left\{\lambda_{1}^{o}\left[D\left(x, y, T_{i}\right)-T_{V}\right]+k g(|\nabla I|)-\nabla g \cdot \vec{N}\right\} \vec{N}\right]=0 \quad$ for $(x, y) \in \overline{C\left(q, t, T_{i}\right)}$

Then we have $\lambda_{1}^{*}\left(T_{i}\right) \geq \max \left(\lambda_{1}^{o}, 0\right)$. To simplify computations, we approximate $\max \left(\lambda_{1}^{o}, 0\right)$ by $\lambda_{1}^{C}\left(T_{i}\right)$ and

thus have

$$
\overline{\lambda_{1}^{*}\left(T_{i}\right)}=\frac{\int_{\lambda_{1}^{c}}^{\infty} \lambda_{1}^{*} \exp \left\{-\frac{\left(\lambda_{1}^{*}-\lambda_{1}^{c}\right)^{2}}{T_{i}}\right\} d \lambda}{\int_{\lambda_{1}^{C}}^{\infty} \exp \left(-\frac{\left(\lambda_{1}^{*}-\lambda_{1}^{C}\right)^{2}}{T_{i}}\right) d \lambda}=\lambda_{1}^{C}\left(T_{i}\right)+\sqrt{\frac{T_{i}}{\pi}}
$$

From Eq. (A-1) and (A-2), $\dot{\mathrm{t}}$ is obvious that $\overline{\sigma^{2}\left(T_{i}\right)} \geq \sigma_{C}^{2}\left(T_{i}\right), \overline{\lambda_{1}^{*}\left(T_{i}\right)} \geq \lambda_{1}^{C}\left(T_{i}\right)$. Let $\sigma_{C}^{2}\left(T_{0}\right)=\sigma_{C\left(q, 0, T_{1}\right)}^{2}$, $\lambda_{1}^{C}\left(T_{0}\right)=\lambda_{1}^{C\left(q, 0, T_{1}\right)}$, and $I_{0}^{C}\left(T_{0}\right)=I_{0}^{C\left(q, 0, T_{1}\right)}$. Please be noted that $T_{0}$ does not exist and the above equations only serve to simplify notations for the following discussions. Since the contour deformations made in Step1 are small, the differences between $\sigma_{C}^{2}\left(T_{i}\right)$ and $\sigma_{C}^{2}\left(T_{i-1}\right), \lambda_{1}^{C}\left(T_{i}\right)$ and $\lambda_{1}^{C}\left(T_{i-1}\right)$ can be ignored. In Eq. (A-1) and $\quad(\mathrm{A}-2), \quad$ as $\quad T_{i} \rightarrow 0, \quad \overline{\lambda_{1}^{*}\left(T_{i}\right)} \rightarrow \lambda_{1}^{C}\left(T_{i}\right) \approx \lambda_{1}^{C}\left(T_{i-1}\right), \quad \overline{\sigma^{2}\left(T_{i}\right)} \rightarrow \sigma_{C}^{2}\left(T_{i}\right) \approx \sigma_{C}^{2}\left(T_{i-1}\right)$, $\overline{I_{0}\left(T_{i}\right)}=I_{0}^{C}\left(T_{i}\right) \approx I_{0}^{C}\left(T_{i-1}\right), \overline{C\left(q, t, T_{i+1}\right)}$ computed according to Eq. (24) either will stop at $\overline{C\left(q, t, T_{i}\right)}$ (for $i=1$ ) or may even move backward (for $i>1$ ) therefore $\overline{\Omega_{C}\left(t, T_{i+1}\right)} \subseteq \Omega_{\Gamma}$ is guaranteed to be satisfied. By a careful selection of $T_{i}, \overline{C\left(q, t, T_{i+1}\right)}$ can move outward and approach target boundary while satisfy $\overline{\Omega_{C}\left(t, T_{i+1}\right)} \subseteq \Omega_{\Gamma}$. With $\overline{\Omega_{C}\left(t, T_{i+1}\right)} \subseteq \Omega_{\Gamma}$, we can then repeat the above procedures for computing $\overline{\lambda_{1}^{*}\left(T_{i+1}\right)}$, $\overline{\sigma^{2}\left(T_{i+1}\right)}, \overline{I_{0}\left(T_{i+1}\right)}$ and let $i=i+1$ until $i$ equals $n$. 


\section{Reference}

[1] X. Wang, L. He, and W. Wee, "Constrained Optimization: A Geodesic Snake Approach", vol. II, pp.77-80, IEEE ICIP, 2002

[2] M. Kass, A. Witkin and D. Terzopoulos, Snakes: Active Contour Models, IJCV, 1 (4) pp. 321-331, 1998.

[3] R. Malladi, J. Sethian and B. Vemuri, Shape Modeling with Front Propagation, IEEE Trans on PAMI, 17 (2) pp. 158-171, 1995.

[4] V. Casellas, R. Kimmel and G. Sapiro, Geodesic Active Contours, IJCV, 22 (1) pp. 61-79, 1997.

[5] X. Wang, L. He, and W. Wee, "A Constrained Optimization Approach to Deformable Contour Method", pp. 183-192, British Machine Vision Conference, 2002.

[6] T. Chan and L. Vese, Active Contours without Edges, IEEE Trans on Image Processing, 10 (2) pp. 266-277, 2001.

[7] C. Samson, L. Blanc-Feraud, G. Aubert and J. Zerubia, A Level Set Model for Image Classification, IJCV, 40 (3) 187-197, 2000.

[8] N. Paragios and R. Deriche, Geodesic Active Contours and Level Set Methods for Supervised Texture Segmentation, IJCV, Mar. 2002.

[9] D. Geiger, Girosi, F., Parallel and deterministic algorithms from MRFs: surface reconstruction IEEE Trans. on PAMI, 13 (5), pp. 401 - 412, 1991.

[10] K. Siddiqi, Y. B. Lauziere, A. Tannenbaum, S. W. Zucker, Area and length minimizing flows for shape segmentation, IEEE Trans. on Image Processing, Vol. 7 pp 433-443, 1998.

[11] T. McInerney and D. Terzopoulos, Topology adaptive deformable surfaces for medical image volume segmentation. IEEE Trans. on Medical Imaging, Vol. 18, No.10, pp. 840-850, 1999.

[12] C. Xu, and J. Prince, Snakes, shape, and gradient vector flow. IEEE Trans. on Image Processing, Vol. 7, pp. 359-369, 1998. 
[13] L. Cohen, On active contour models and balloons, CVGIP: Image Understanding. Vol. 52, No.2, pp. 211-218, March, 1991.

[14] Stovik, G. 1994. "A Bayesian approach to dynamic contours through stochastic sampling and simulated annealing". IEEE Trans. on PAMI, Vol.16, No.10, pp. 976-986.

[15] Grzeszczuk, R. and Levin, D. 1997. ““'Brownian String”, Segmenting images with stochastically deformable contours". IEEE Trans. on PAMI, Vol. 19, No. 10, pp. 1100-1114.

[16] X. Wang, L. He, and William G. Wee, "A Constrained Optimization Approach to Deformable Contour Method", IJCV (in press) (can also be found in www.ececs.uc.edu/ xwang)

[17] Tsai A, Zhang J, Willsky A, 'Expectation-Maximization Algorithms for Image Processing Using Multiscale Methods and Mean Field Theory, with Applications to Laser Radar Range Profiling and Segmentation", Optical Engineering, vol. 40, no. 7, pp. 1287-1301, July 2001.

[18] N. Paragios and M. Rousson, "Shape Priors for Level Set Representations", Proceedings of European Conference in Computer Vision, (ECCV 2002) June 2002.

[19] D. Cremers, T. Kohlberger, and C. Schnorr, "Nonlinear Shape Statistics in Mumford-Shah Based Segmentation,” pp. 93-109, ECCV 2002.

[20] X. Wang, F. Gao, Z. Peng, L. He, and W. Wee, “An Integrated Approach to the Segmentation and Recognition of Objects using Thin Plate Spline Method", International Conference of Vision Interfaces 2003 .

[21] T. Hofmann, J. Puzicha, J. M., Buhmann, "Unsupervised texture segmentation in a deterministic annealing framework", IEEE Trans on PAMI, pp. 803-818, Vol. 20 No. 8 , Aug. 1998.

[22] Yuri Boykov, Olga Veksler and Ramin Zabih, "Fast Approximate Energy Minimization via Graph Cuts”, IEEE Trans. on PAMI, Vol. 23, No. 11, Nov. 2001.

[23] S. Geman and D. Geman, "Stochastic Relaxation, Gibbs Distribution, and the Bayesian Restoration of Images”, IEEE Trans. on PAMI, Vol. 6, pp 721 - 741, 1984. 
[24] D. Greig, B. Porteous, and A. Seheult, "Exact Maximum: A Posteriori Estimation for Binary Images,” J. Royal Statistical Soc., Series B, Vol. 51, No. 2, pp. 271-279, 1989.

[25] Yuri Boykov and Marie-Pierre Jolly, "Interactive Graph Cuts for Optimal Boundary and Region Segmentation of Objects in N-D images”, ICCV, Pages I: 105-112, 2001.

[26] T. Hofmann and J. M. Buhmann, 'Pairwise data clustering by deterministic annealing", IEEE Transactions on PAMI, Vol. 19, No. 1, pp 1- 14, Jan. 1997.

[27] J. Tohka, "Surface extraction from volumetric images using deformable meshes: a comparative study," the seventh European Conference on Computer Vision, pp. 350-364, 2002.

[28] L. He, B. Everding, Z. Peng, X. Wang, C.Y. Han, K. Weiss, and W.G.Wee, "A Comparative Survey of Deformable Contour Methods on Medical Image Segmentation”, IEEE Trans. on Medical Imaging, (under second round review).

[29] A. Daniilidis and C. Lemarechal, "Proximal Convexification Procedures in Combinatorial Optimization”, INRIA Research Report RR 4550, September 2002.
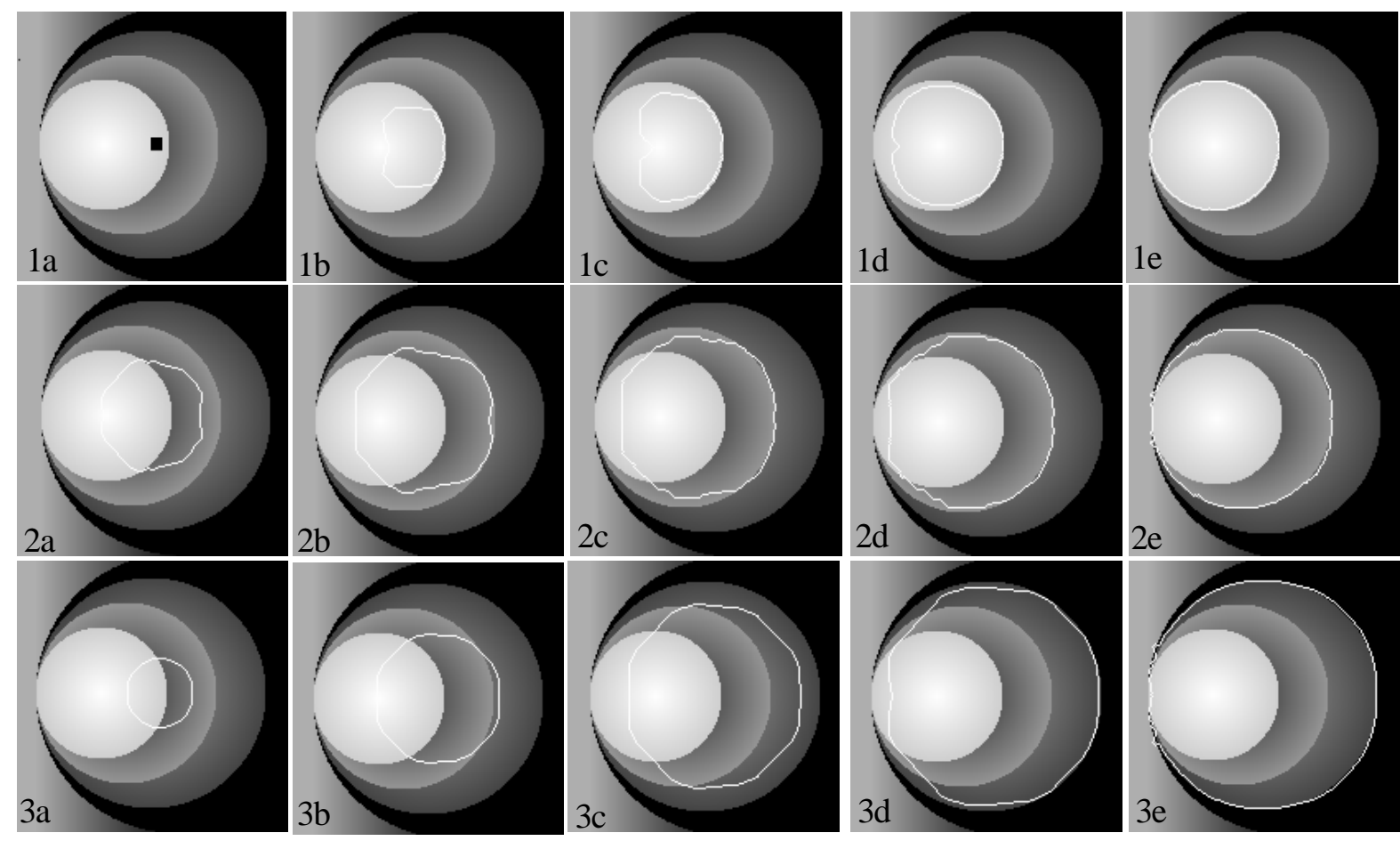

Fig. 6.1b to 6.1e illustrate the contour evolution process of extracting $M C_{1}$ using the proposed method. Fig. 6.2a to 6.2e, and Fig. 6.3a to 6.3e illustrate the contour evolution process of extracting $M C_{2}$ and $M C_{3}$ respectively using the proposed method 

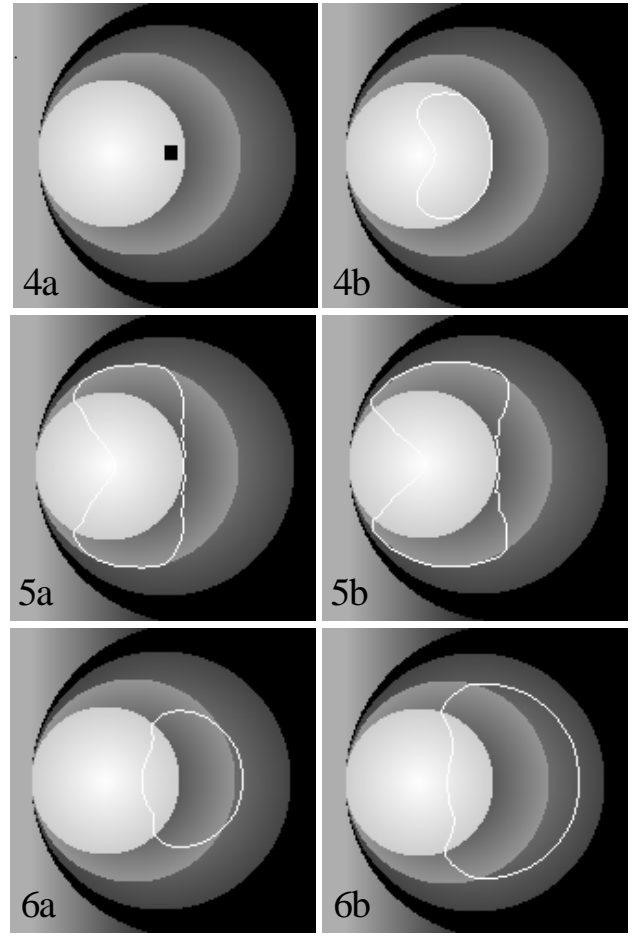
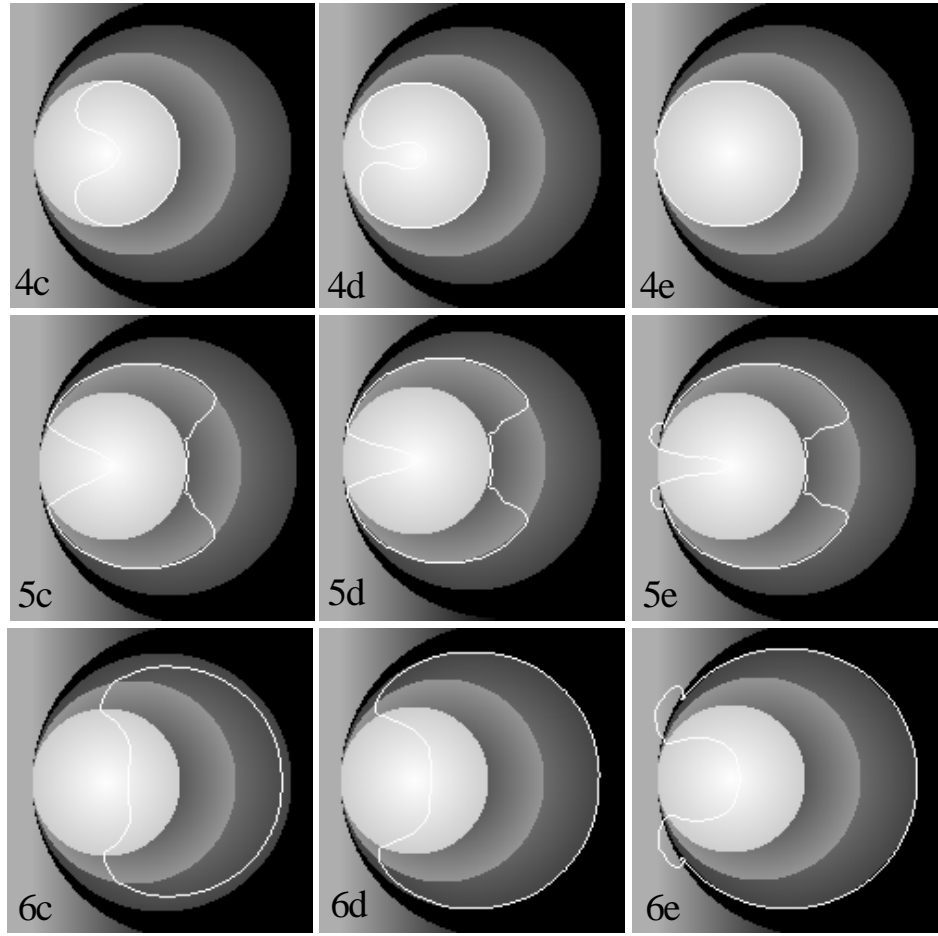

Fig. $6.4 \mathrm{~b}$ to $6.4 \mathrm{e}$ illustrate the contour evolution process of extracting $M C_{1}$ using Eq. (17). Fig. 6.5a to $6.5 \mathrm{e}$, and Fig. 6.6a to 6.6e illustrate the contour evolution process of extracting $M C_{2}$ and $M C_{3}$ respectively using Eq. (17)
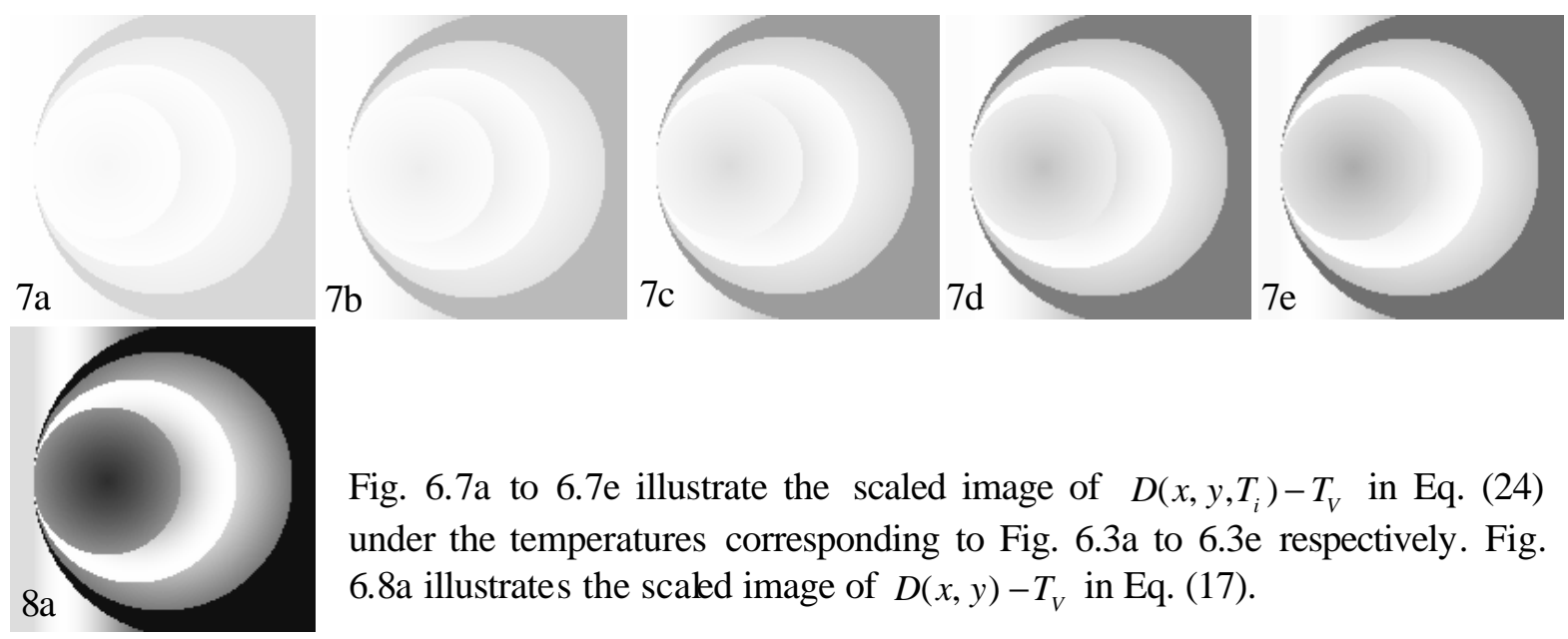

Fig. 6.7a to 6.7e illustrate the scaled image of $D\left(x, y, T_{i}\right)-T_{V}$ in Eq. (24) under the temperatures corresponding to Fig. 6.3a to 6.3e respectively. Fig. 6.8a illustrates the scaled image of $D(x, y)-T_{V}$ in Eq. (17). 


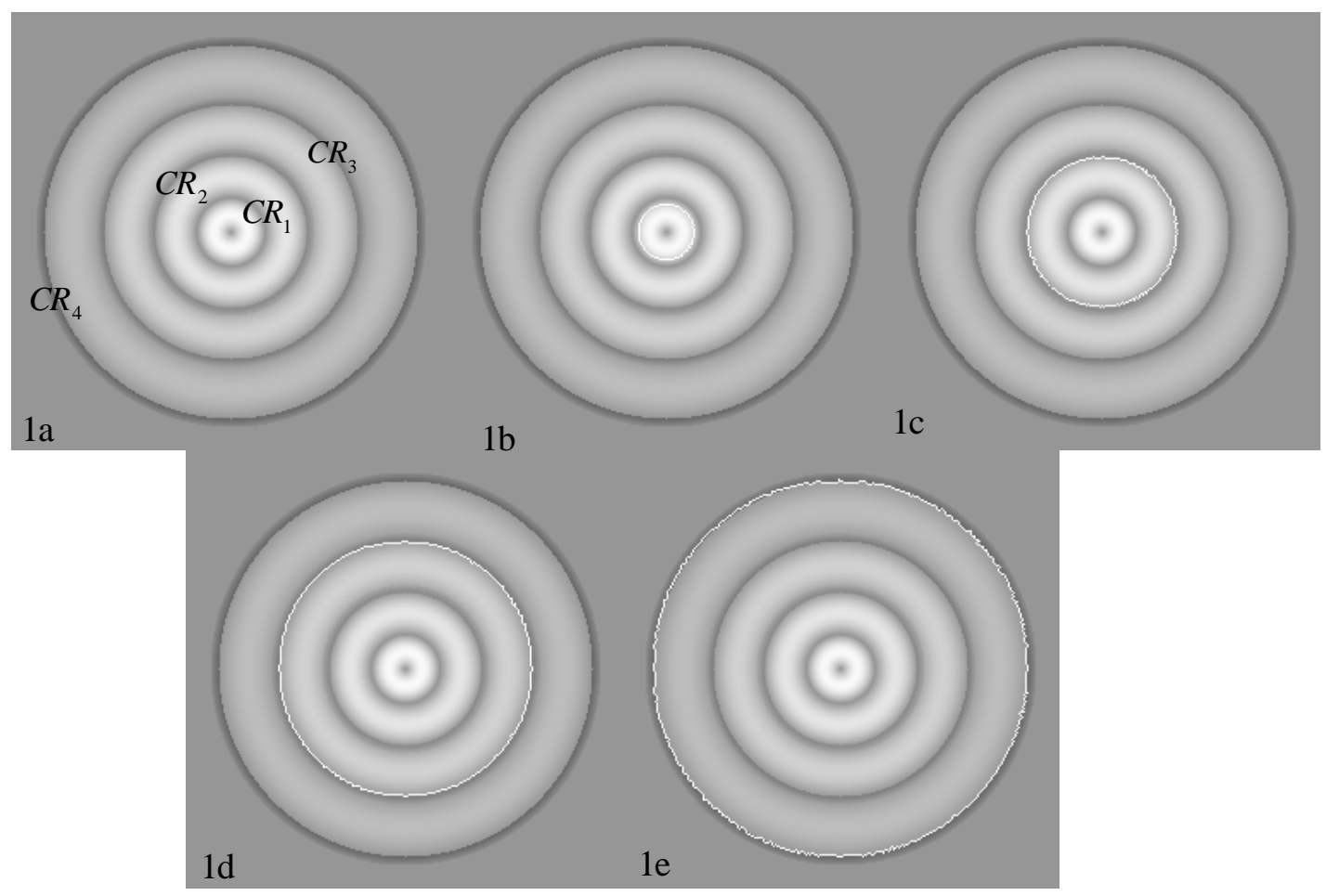

Fig. 7.1a The test image for different settings of $T_{V}$. Fig. 7.1b The resulting contour with $T_{V}=0.35$, Fig. 7.1c The resulting contour with $T_{V}=0.1$, Fig. 7.1d The resulting contour with $T_{V}=0.03$, Fig. $7.1 \mathrm{e}$ The resulting contour with $T_{V}=0.0001$.
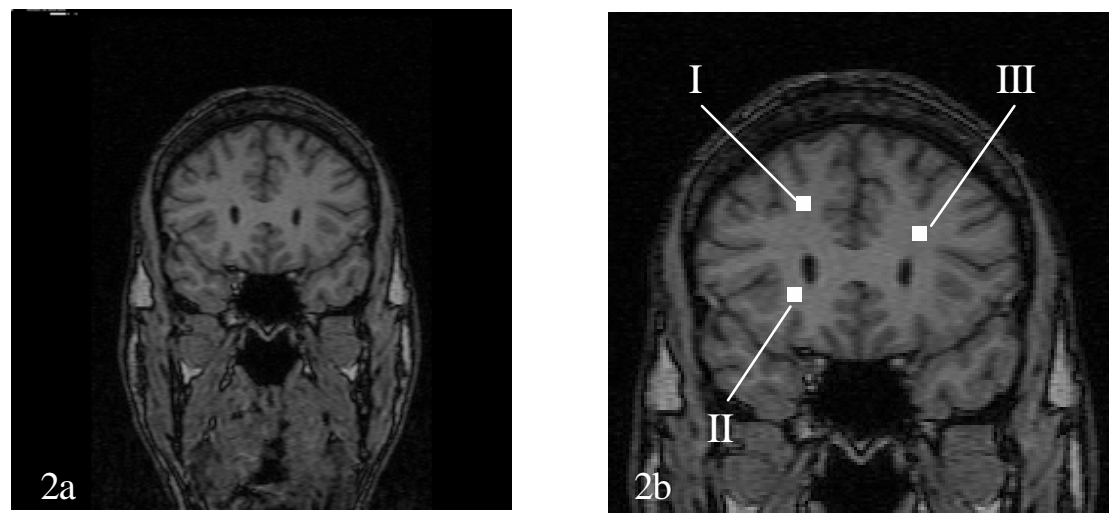

Fig. 7.2a Test image for parameter sensitivity experiments. Fig. 7.2b Zoomed image of the brain and the positions of the initial locations I, II, and III. 

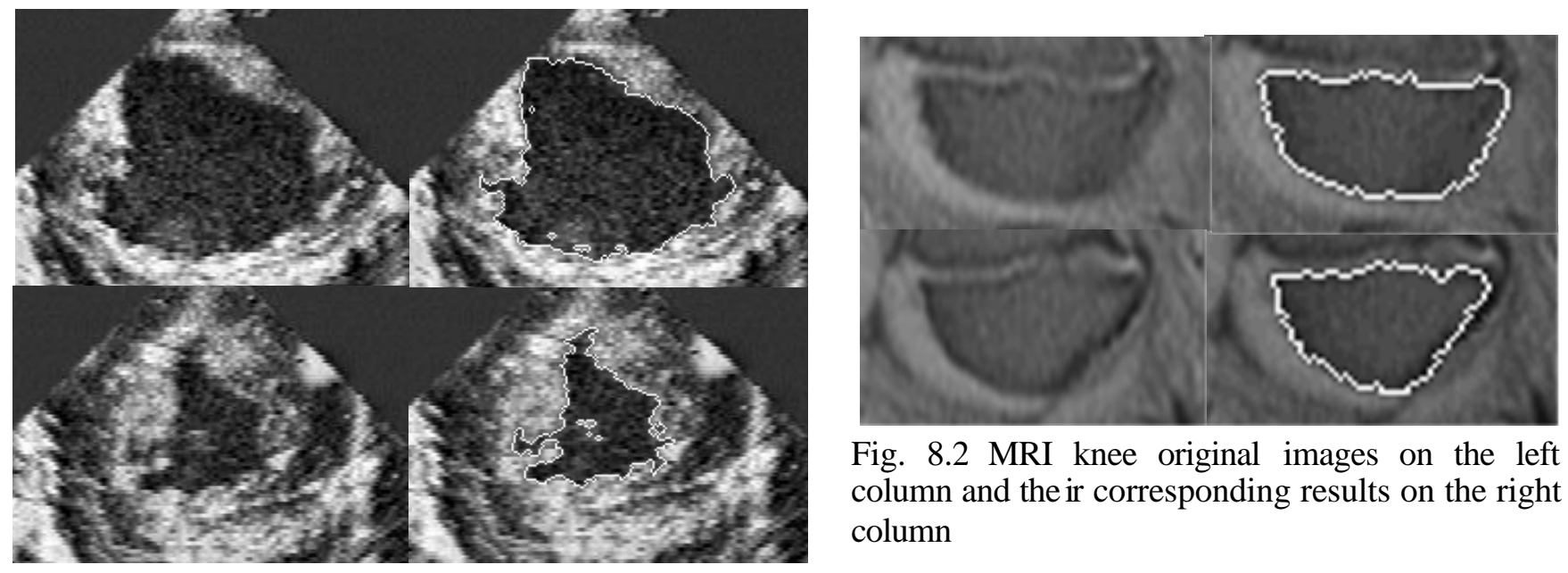

Fig. 8.2 MRI knee original images on the left column and the ir corresponding results on the right column

Fig. 8.1 Ultrasound pig heart original images in the left column and the ir corresponding results in the right column

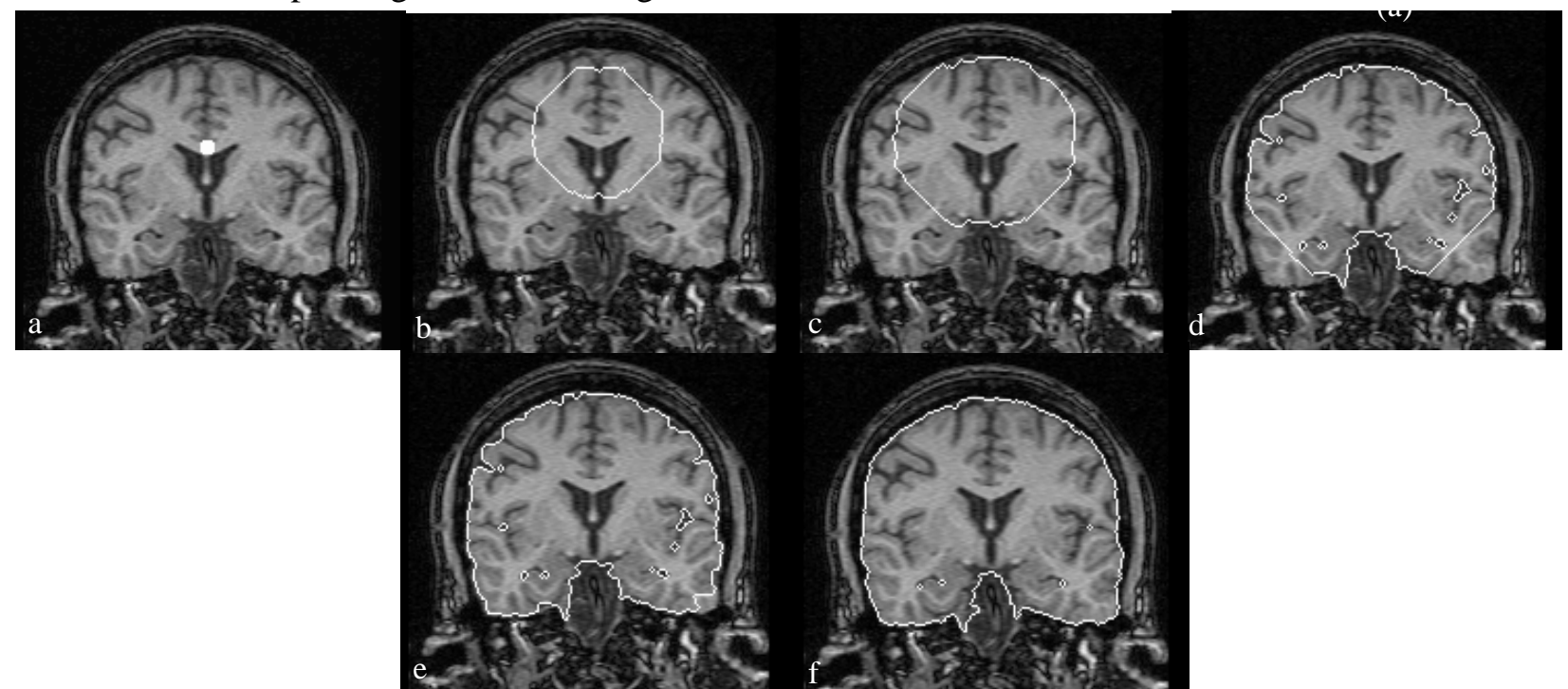

Fig. 8.3 (a) Original MRI brain mage (white dot indicates the position of the initial contour). (b) to (g) The extraction process of the exterior boundary of intracranial
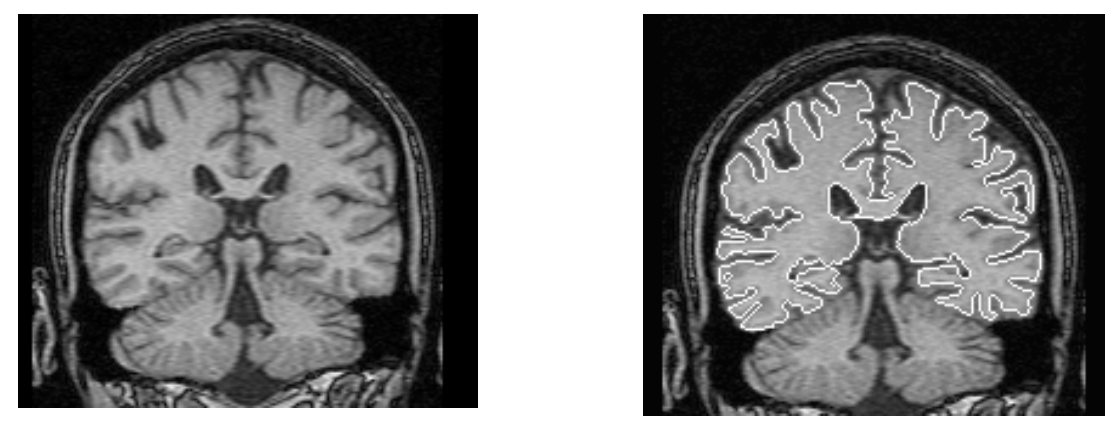

Fig. 8.4 MRI brain image two and the result 

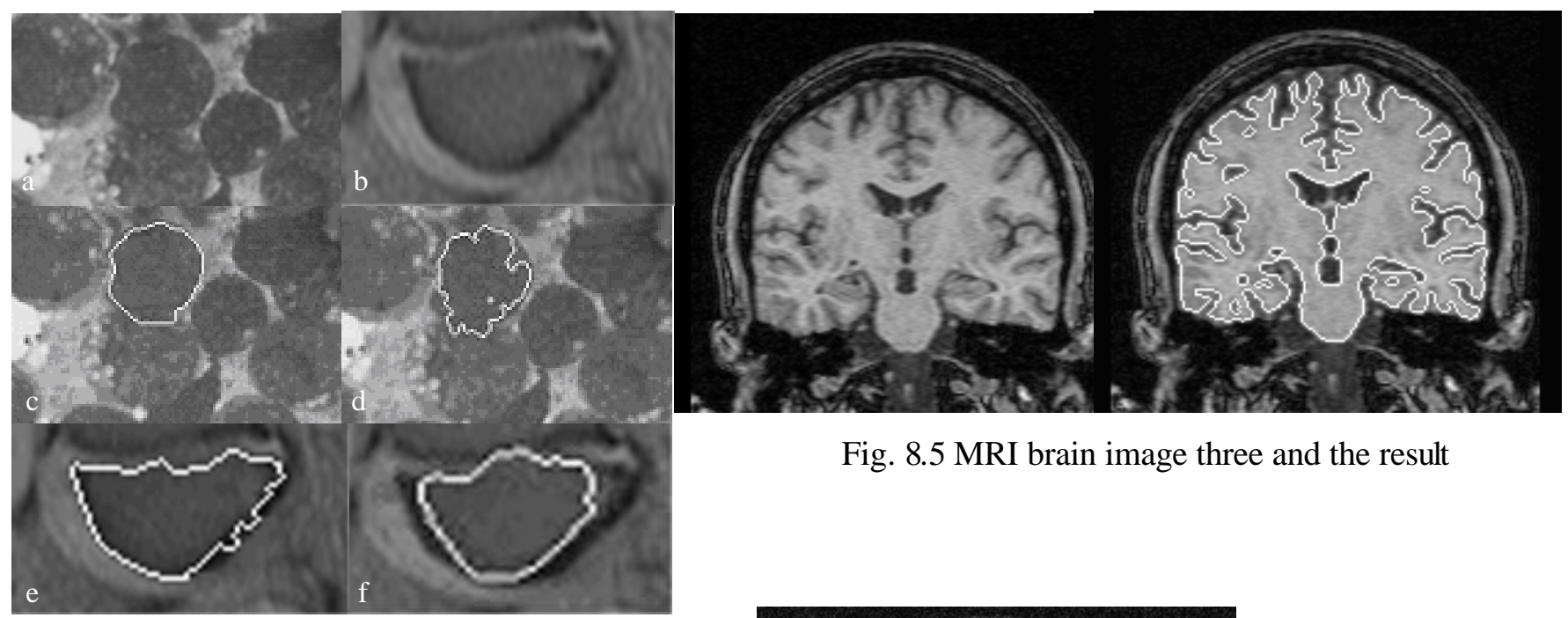

Fig. 8.5 MRI brain image three and the result

Fig. 8.6 Comparison results with CODCM [16]. (c) and (e) are segmentation results using the proposed method. (d) and (f) are segmentation results using CODCM [16].

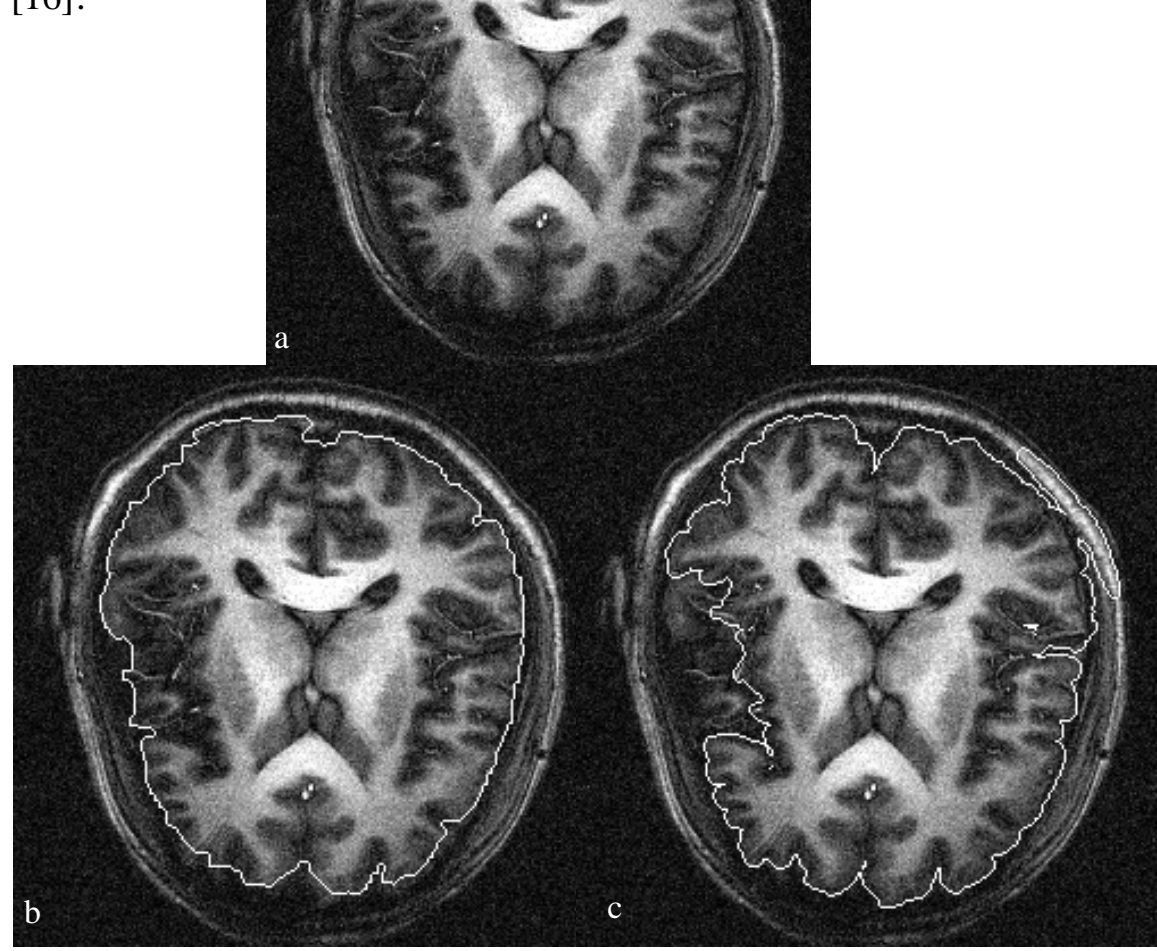

Fig. 8.7 Comparison results with CODCM [16]. (b) is the segmentation result of the external boundary of intracranial using the proposed method. (c) is the segmentation result of the external boundary of intracranial using CODCM [16]. 

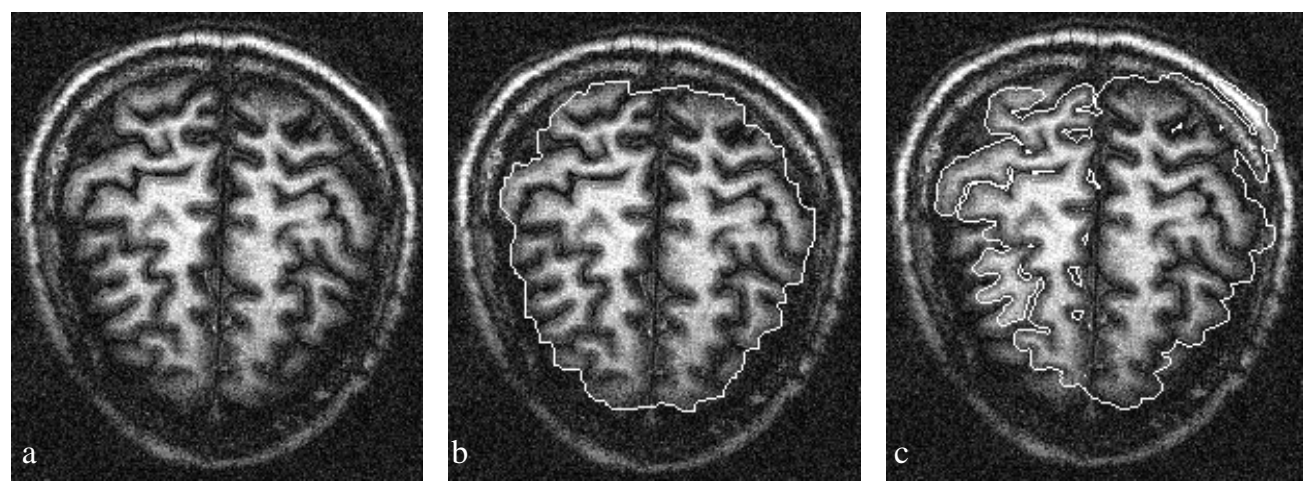

Fig. 8.8 Comparison results with CODCM [16]. (b) is the segmentation result of the external boundary of intracranial using the proposed method. (c) is the segmentation result of the external boundary of intracranial using CODCM [16].
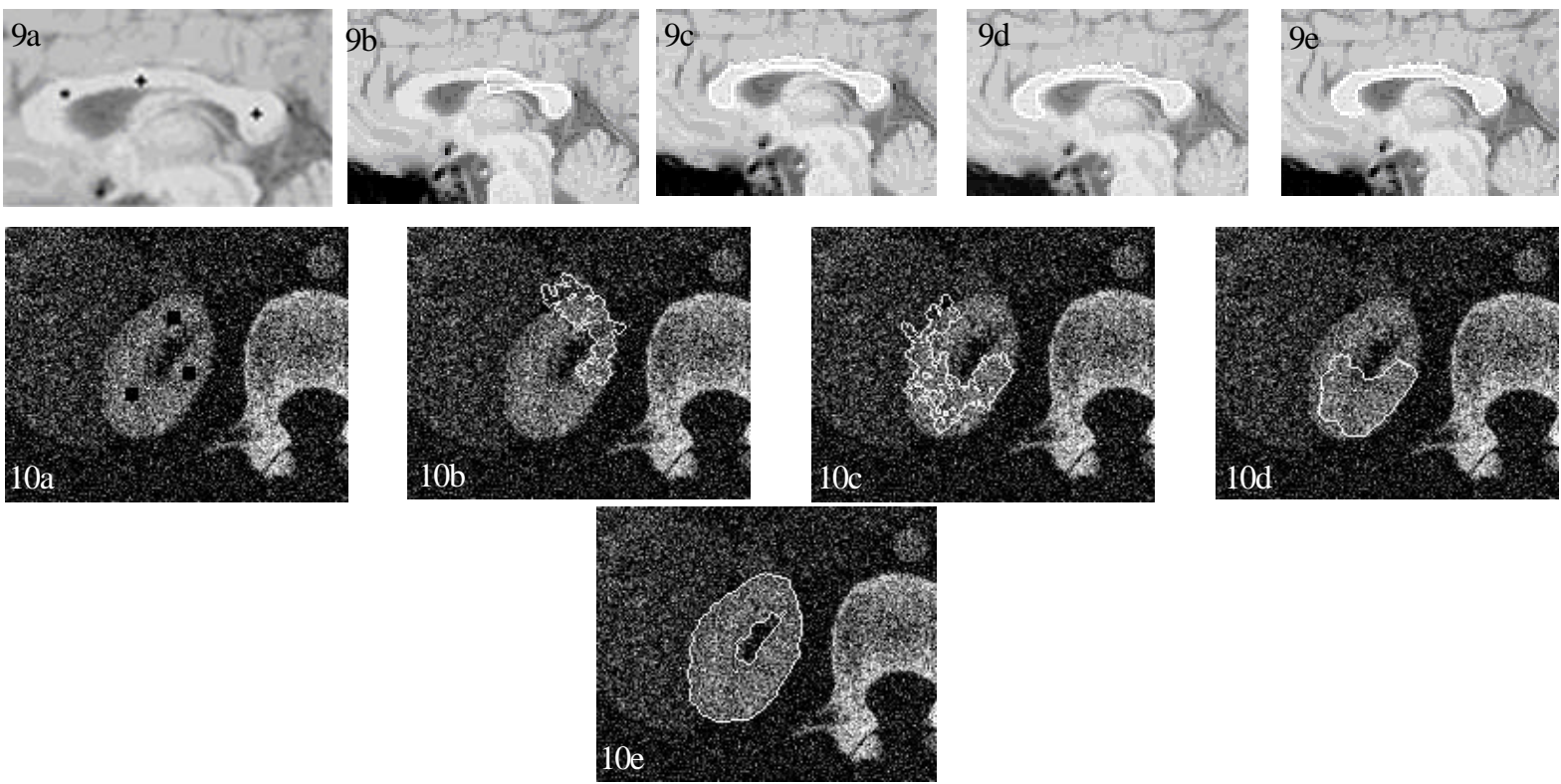

Fig. 8.9a, 10a Original images of the comparison, 9b, 10b The results of T-snake, 9c, 10c The results of godesic snake, $9 \mathrm{~d}, 10 \mathrm{~d}$ The results of Area-length snake, 9e, 10e The results of proposed method. 